\title{
Saddle-Node Heteroclinic Orbit and Exact Nontraveling Wave Solutions for (2+1)D KdV-Burgers Equation
}

\author{
Da-Quan Xian \\ School of Sciences, Southwest University of Science and Technology, Mianyang 621010, China \\ Correspondence should be addressed to Da-Quan Xian; daquanxian@163.com
}

Received 21 September 2012; Accepted 8 December 2012

Academic Editor: de Dai

Copyright (C) 2013 Da-Quan Xian. This is an open access article distributed under the Creative Commons Attribution License, which permits unrestricted use, distribution, and reproduction in any medium, provided the original work is properly cited.

\begin{abstract}
We have undertaken the fact that the periodic solution of $(2+1) \mathrm{D}$ KdV-Burgers equation does not exist. The Saddle-node heteroclinic orbit has been obtained. Using the Lie group method, we get two-(1+1)-dimensional PDE, through symmetric reduction; and by the direct integral method, spread F-expansion method, and $\left(G^{\prime} / G\right)$-expansion method, we obtain exact nontraveling wave solutions, for the (2+1)D KdV Burgers equation, and find out some new strange phenomenons of sympathetic vibration to evolution of nontraveling wave.
\end{abstract}

\section{Introduction}

We consider the (2+1)-dimensional Korteweg-de Vries Burgers $((2+1) \mathrm{D}$ KdV Burgers) equation

$$
\left(u_{t}+u u_{x}-\beta u_{x x}+\alpha u_{x x x}\right)_{x}+\gamma u_{y y}=0,
$$

where $u: R_{x} \times R_{y} \times R_{t}^{+} \rightarrow R, \alpha, \beta$, and $\gamma$ are real parameters. Equation (1) is model equation for wide class of nonlinear wave models in an elastic tube, liquid with small bubbles, and turbulence [1-3]. Much attention has been put on the study of their exact solutions by some methods [4], such as, a complex line soliton by extended tanh method with symbolic computation [5], exact traveling wave solutions including solitary wave solutions, periodic wave and shock wave solutions by extended mapping method, and homotopy perturbation method $[6,7]$.

It is well known that the investigation of exact solutions of nonlinear evolution equations plays an important role in the study of nonlinear physical phenomena. Many effective methods have been presented [7-22], such as functional variable separation method $[8,9]$, homotopy perturbation method [12], F-expansion method [7, 13], Lie group method $[14,15]$, variational iteration method [16], homoclinic test method [17-19], Exp-function method [20, 21], and homogeneous balance method [22]. Practically, there is no unified method that can be used to handle all types of nonlinearity.
In this paper, we will discuss the existence of periodic traveling wave solution and seek the Saddle-Node heteroclinic orbit, and further use the Lie group method with the aid of the symbolic computation system Maple to construct the non-traveling wave solutions for (1).

\section{Existence of Periodic Traveling Wave Solution of (1)}

Introducing traveling wave transformation in this form

$$
u(x, y, t)=u(\xi), \quad \xi=p x+q y-c t
$$

permits us to convert (1) into an ODE for $u=u(\xi)$

$$
p\left(p u u_{\xi}-\beta p^{2} u_{\xi \xi}+\alpha p^{3} u_{\xi \xi \xi}\right)_{\xi}-r u_{\xi \xi}=0
$$

where $r=p c-q^{2} \gamma$, Integrating (3) with respect to $\xi$ twice and taking integration constant to $A$ yields

$$
2 \alpha p^{4} u_{\xi \xi}-2 \beta p^{3} u_{\xi}+p^{2} u^{2}-2 r u=A .
$$

Letting $u_{\xi}=v$, thus nonlinear ordinary differential equation (4) is equivalent to the autonomous dynamic system as follows:

$$
\begin{gathered}
\frac{d u}{d \xi}=v, \\
\frac{d v}{d \xi}=\frac{1}{2 \alpha p^{4}}\left(2 \beta p^{3} v-p^{2} u^{2}+2 r u+A\right) .
\end{gathered}
$$


The dynamic system (5) has two balance points:

$$
\begin{aligned}
& P_{1}\left(u_{1}, v_{1}\right)=\left(\frac{r+\sqrt{r^{2}+p^{2} A}}{p^{2}}, 0\right), \\
& P_{2}\left(u_{2}, v_{2}\right)=\left(\frac{r-\sqrt{r^{2}+p^{2} A}}{p^{2}}, 0\right) .
\end{aligned}
$$

The Jacobi matrixes at the balance points for the right-hand side of (5) are obtained as follows, respectively:

$$
\begin{aligned}
& J_{1}=\left(\begin{array}{cc}
0 & 1 \\
-\frac{\sqrt{r^{2}+p^{2} A}}{p^{4} \alpha} & \frac{\beta}{p \alpha}
\end{array}\right), \\
& J_{2}=\left(\begin{array}{cc}
0 & 1 \\
\frac{\sqrt{r^{2}+p^{2} A}}{p^{4} \alpha} & \frac{\beta}{p \alpha}
\end{array}\right) .
\end{aligned}
$$

Their latent equations are expressed, respectively, as,

$$
\begin{aligned}
& p^{3} \lambda(p \alpha \lambda-\beta)+\sqrt{r^{2}+p^{2} A}=0, \\
& p^{3} \lambda(p \alpha \lambda-\beta)-\sqrt{r^{2}+p^{2} A}=0 .
\end{aligned}
$$

Relevant latent roots are as follows respectively:

$$
\begin{aligned}
& \lambda_{1}=\frac{p \beta \pm \sqrt{p^{2} \beta^{2}-4 \alpha \sqrt{r^{2}+p^{2} A}}}{2 p^{2} \alpha} \\
& \lambda_{2}=\frac{p \beta \pm \sqrt{p^{2} \beta^{2}+4 \alpha \sqrt{r^{2}+p^{2} A}}}{2 p^{2} \alpha} .
\end{aligned}
$$

Obviously, if $p^{2} \beta^{2}>4 \alpha \sqrt{r^{2}+p^{2} A}$, then $\lambda_{1}$ are two positive real roots, therefore $P_{1}$ is a nonsteady node point. If $0<$ $p^{2} \beta^{2}<4 \alpha \sqrt{r^{2}+p^{2} A}$, then $\lambda_{1}$ are conjugate complex roots and real part is positive, so $P_{1}$ is a nonsteady focus point. And $\lambda_{2}$ is a positive and minus real root, thus $P_{2}$ is a saddle point. From (5), we know the phase trajectory on the phase plane satisfies

$$
\frac{d v}{d u}=\frac{2 \beta p^{3} v-p^{2} u^{2}+2 r u+A}{2 \alpha p^{4} v}
$$

Integrating (11), we can obtain

$$
H(u, v)=A u+r u^{2}-\frac{1}{3} p^{2} u^{3}+2 \beta p^{3} u v-\alpha p^{4} v^{2},
$$

where $H(u, v)$ is a total energy or Hamiliton function of system (4). Apparently

$$
u_{\xi} \neq-\frac{\partial H}{\partial v}, \quad v_{\xi} \neq \frac{\partial H}{\partial u} .
$$

Consequently, the system expressed in (12) is not a conservative one, then periodic traveling wave solution of (1) does not exist.

We conclude the above analysis in the following theorem.

Theorem 1. Under the traveling wave transformation, the periodic solution of (2+1)-dimensional KdV-Burgers equation does not exist.

But, saddle-node heteroclinic orbits and nontraveling periodic solution do exist, which will be discussed later in this paper.

\section{Saddle-Node Heteroclinic Orbits of KdV-Burgers Equation}

First, we assume the solutions of (4) in the form

$$
u(\xi)=\frac{r+\sqrt{r^{2}+p^{2} A}}{p^{2}}+\frac{b}{\left(1+e^{a \xi}\right)^{2}} .
$$

Substituting (14) into (4) yields

$$
\begin{aligned}
& 2\left(4 \alpha p^{4} a^{2}+\sqrt{r^{2}+p^{2} A}+2 \beta p^{3} a\right) e^{2 a \xi} \\
& -4\left(\alpha p^{4} a^{2}-\sqrt{r^{2}+p^{2} A}-\beta p^{3} a\right) e^{a \xi} \\
& +2 \sqrt{r^{2}+p^{2} A}+p^{2} b=0 .
\end{aligned}
$$

Then we get

$$
\begin{gathered}
4 \alpha p^{4} a^{2}+\sqrt{r^{2}+p^{2} A}+2 \beta p^{3} a=0, \\
\alpha p^{4} a^{2}-\sqrt{r^{2}+p^{2} A}-\beta p^{3} a=0 \\
2 \sqrt{r^{2}+p^{2} A}+p^{2} b=0 .
\end{gathered}
$$

Solving the system (16) gets

$$
a=-\frac{\beta}{5 \alpha p}, \quad b=-\frac{12 \beta^{2}}{25 \alpha}, \quad \sqrt{r^{2}+p^{2} A}=\frac{6 p^{2} \beta^{2}}{25 \alpha} .
$$

Substituting (17) into (14) obtains

$$
\begin{aligned}
u(\xi) & =\frac{r+\sqrt{r^{2}+p^{2} A}}{p^{2}}-\frac{12 \beta^{2}}{25 \alpha} \frac{1}{\left(1+e^{-(\beta / 5 \alpha p) \xi}\right)^{2}} \\
& =u_{1}-\frac{3 \beta^{2}}{25 \alpha}\left(1+\tanh \frac{\beta}{20 \alpha} \xi\right)^{2} .
\end{aligned}
$$

Evidently, $\xi \rightarrow-\infty \Rightarrow u(\xi) \rightarrow u_{1}, \xi \rightarrow+\infty \Rightarrow u(\xi) \rightarrow$ $u_{1}-\left(6 \beta^{2} / 25 \alpha\right)=u_{2}$. Thus (18) is a saddle-node heteroclinic orbit through nonsteady node point $P_{1}$ and saddle point $P_{2}$ [23]. 

obtain

Ecumenic, taking the Hamiliton function $H(u, v)=B$, we

$$
\begin{aligned}
\frac{d u}{d \xi} & =v \\
& =\frac{3 p \beta u \pm \sqrt{3 u\left[3 A \alpha+3\left(p^{2} \beta^{2}+r \alpha\right) u-p^{2} \alpha u^{2}\right]-9 B \alpha}}{3 \alpha p^{2}}
\end{aligned}
$$

where $B$ is an arbitrary constant. Integrating (19) with respect to $\xi$ we have

$$
\begin{aligned}
& \int^{u(\xi)} \frac{3 \alpha p^{2}}{3 p \beta s \pm \sqrt{3 s\left[3 A \alpha+3\left(p^{2} \beta^{2}+r \alpha\right) s-p^{2} \alpha s^{2}\right]-9 B \alpha}} d s \\
& =\xi+\xi_{0},
\end{aligned}
$$

where $\xi_{0}$ is an arbitrary constant. We can see that (4) has the general solution (20) and all partial cases as include above result can be found from the general solution of (20). Example, take $\alpha \sqrt{r^{2}+p^{2} A}-p^{2} \beta^{2}=0,3 B \alpha+A \beta^{2}=0$, $r \alpha+p^{2} \beta^{2}=0$ in (20), we find a solution of (4) as follows:

$$
u(\xi)=-\frac{3 \beta^{2}}{4 \alpha}\left[1+\tanh \left(\frac{\beta}{4 p \alpha} \xi+\xi_{0}\right)\right]^{2} .
$$

It is a heteroclinic orbit too.

\section{Li Symmetry of (1)}

This section devotes to Li symmetry of $(1)[14,15]$. Let

$$
\sigma=\sigma\left(x, y, t, u, u_{t}, u_{x}, u_{y}, \ldots\right) .
$$

be the Li symmetry of (1). From Lie group theory, $\sigma$ satisfies the following equation

$$
\sigma_{x t}+2 u_{x} \sigma_{x}+u \sigma_{x x}+\sigma u_{x x}-\beta \sigma_{x^{3}}+\alpha \sigma_{x^{4}}+\gamma \sigma_{y y}=0 .
$$

We take the function $\sigma$ in the form

$$
\sigma=a_{1} u_{x}+a_{2} u_{y}+a_{3} u_{t}+a_{4} u+a_{5}
$$

where $a_{i}=a_{i}(x, y, t): R_{x} \times R_{y} \times R_{t}^{+} \rightarrow R(i=1, \ldots, 5)$ are functions to be determined later. Substituting (3) into (2) yields

$$
\begin{gathered}
a_{1}=-\frac{1}{2 \gamma} k_{2}^{\prime}(t) y+k_{1}(t), \quad a_{2}=k_{2}(t), \\
a_{3}=c, \quad a_{4}=0, \quad a_{5}=\frac{1}{2 \gamma} k_{2}^{\prime \prime}(t) y-k_{1}^{\prime}(t),
\end{gathered}
$$

where $k_{j}(t)(j=1,2)$ are arbitrary functions of $t, c$ is an arbitrary constant. Substituting (25) into (24), we obtain the Li symmetries of (1) as follows:

$$
\begin{aligned}
\sigma= & {\left[-\frac{1}{2 \gamma} k_{2}^{\prime}(t) y+k_{1}(t)\right] u_{x}+k_{2}(t) u_{y} } \\
& +c u_{t}+\frac{1}{2 \gamma} k_{2}^{\prime \prime}(t) y-k_{1}^{\prime}(t) .
\end{aligned}
$$

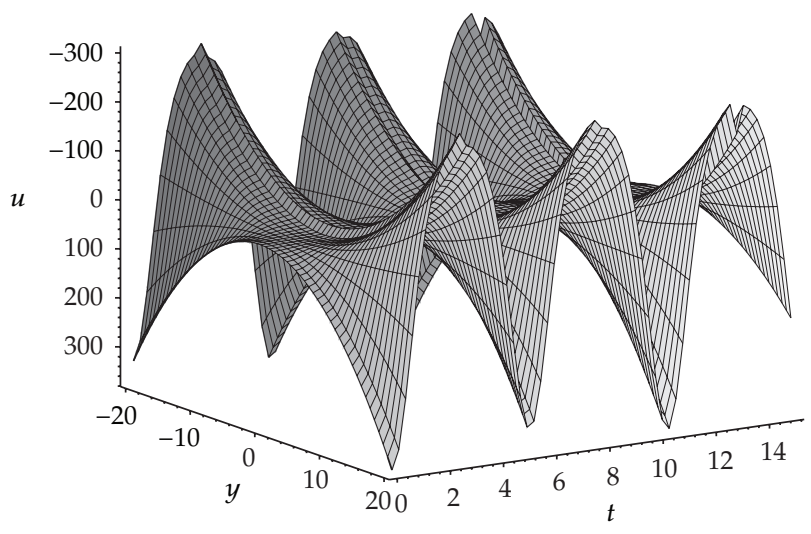

FIGURE 1: The strange phenomenon which is a sympathetic vibration of periodicity on the $t$-axis and paraboloid on $y$-axis for $u_{1}(x, y, t)$ as $x=1$.

\section{Symmetry Reduction and Solutions of (1)}

Based on the integrability of reduced equation of symmetry (26), we are to consider the following three cases.

Case 1. Taking $k_{2}(t)=0$ and $c=0$ in (26) yields

$$
\sigma=k_{1}(t) u_{x}-k_{1}^{\prime}(t)
$$

The solution of the differential equation $\sigma=0$ is

$$
u=\frac{k_{1}^{\prime}(t)}{k_{1}(t)} x+F(y, t), \quad F(y, t): R_{y} \times R_{t}^{+} \rightarrow R .
$$

Substituting (28) into (1) yields the function $F(y, t)$ which satisfies the following linear PDE:

$$
\frac{k_{1}^{\prime \prime}}{k_{1}}+\gamma \frac{\partial^{2} F}{\partial y^{2}}=0
$$

By integrating both sides, we find out the following result:

$$
F(y, t)=-\frac{k_{1}^{\prime \prime}}{2 \gamma k_{1}} y^{2}+k_{3}(t) y+k_{4}(t),
$$

where $k_{3}(t), k_{4}(t)$ are new arbitrary functions of $t$. Substituting (30) into (28), we can get the solutions of (1) as follows:

$$
u_{1}(x, y, t)=\frac{k_{1}^{\prime}(t)}{k_{1}(t)} x-\frac{k_{1}^{\prime \prime}}{2 \gamma k_{1}} y^{2}+k_{3}(t) y+k_{4}(t) .
$$

(1) Given $k_{i}(t)=\operatorname{cn}(t, 0.95)(i=1,3,4), x=1, \gamma=0.6$ in (31), the local structure of $u_{1}$ is obtained (Figure 1). Where $\mathrm{cn}(t, 0.95)$ is an Jacobian elliptic cosine function.

(2) Given $k_{1}(t)=\operatorname{sech}(t), k_{3}(t)=\sin (t), k_{4}(t)=\operatorname{cn}(t$, $0.1), y=1, \gamma=0.6 \mathrm{in}(31)$, the local structure of $u_{1}$ is obtained (Figure 2).

Case 2. Take $k_{1}(t)=t, k_{2}(t)=1$ and $c=0$ in (26), then

$$
\sigma=t u_{x}+u_{y}-1
$$




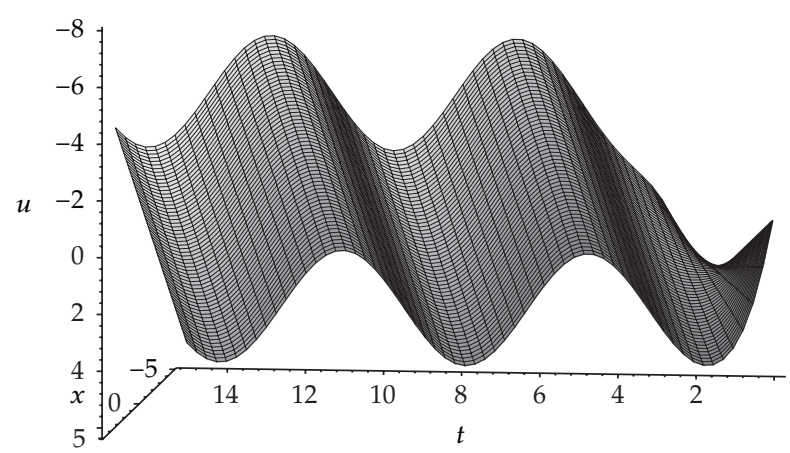

FIGURE 2: The periodic solution which is a periodic nontraveling wave traveling on the $t$-axis for $u_{1}(x, y, t)$ as $y=1$.

Solving the differential equation $\sigma=0$, we can get

$$
u=y+F(t, \xi), \quad \xi=x-t y .
$$

Substituting (33) into (1) and integrating once with respect to $\xi$ yield

$$
F_{t}+F F_{\xi}+\gamma t^{2} F_{\xi}-\beta F_{\xi \xi}+\alpha F_{\xi \xi \xi}=0 .
$$

Again, further using the transformation of dependent variable to (34),

$$
F(t, \xi)=F(\theta), \quad \theta=k\left(t-\frac{1}{3} \gamma t^{3}+\xi\right) .
$$

Substituting (35) into (34) and integrating once with respect to $\theta$ yield

$$
2 k^{2} \alpha F^{\prime \prime}-2 k \beta F^{\prime}+F^{2}+2 F+A=0,
$$

where $A$ is an integration constant, $F^{\prime}=d F(\theta) / d \theta$. We assume that the solution of (36) can be expressed in the form

$$
F(\theta)=a_{0}+a_{1} w(\theta)+a_{2} w(\theta)^{2},
$$

where $a_{i}(i=0,1,2)$ are constants to be determined later, $w(\theta)$ satisfies the following auxiliary equation

$$
w^{\prime}=p+q w^{2} .
$$

Substituting (37) and (38) into (36) and equating the coefficients of all powers of $w$ to zero yield a set of algebra equations for $a_{0}, a_{1}, a_{2}$, and $A$ as follows.

$$
\begin{aligned}
& w^{4}: a_{2}\left(a_{2}+12 \alpha k^{2} q^{2}\right)=0, \\
& w^{3}:-4 \beta k a_{2} q+2 a_{1} a_{2}+4 \alpha k^{2} a_{1} q^{2}=0, \\
& w^{2}: a_{1}^{2}+16 \alpha k^{2} a_{2} q p-2 \beta k a_{1} q+2 a_{2}+2 a_{2} a_{0}=0, \\
& w^{1}: 2 a_{1} a_{0}-4 \beta k a_{2} p+2 a_{1}+4 \alpha k^{2} a_{1} q p=0, \\
& w^{0}: 2 a_{0}+A+4 \alpha k^{2} a_{2} p^{2}+a_{0}^{2}-2 \beta k a_{1} p=0 .
\end{aligned}
$$

Solving the system of function equations with the aid of Maple, we obtain

$$
a_{0}=\frac{3 \beta^{2}-25 \alpha}{25 \alpha}, \quad a_{1}=\frac{6 \beta^{2} q}{25 s \alpha}, \quad a_{2}=\frac{3 \beta^{2} q}{25 \alpha p} .
$$

when $k=\beta / 10 s \alpha, p q<0, A=\left(625 \alpha^{2}-36 \beta^{4}\right) / 625 \alpha^{2}$, where $s=\sqrt{-p q}$.

It is known that solutions of (38) are as follows [24]:

$$
w(\theta)=-s \tanh (s \theta), \quad w(\theta)=-s \operatorname{coth}(s \theta) .
$$

Substituting (41), (40), (37), and (35) into (33), we obtain solutions of (1) as follows:

$$
\begin{aligned}
& u_{2}(x, y, t) \\
& =\frac{1}{25 \alpha}\left\{3 \beta^{2}-25 \alpha-3 q \beta^{2}\right. \\
& \times\left[\tanh \left(\frac{\beta}{10 s \alpha}\left(x-t y+t-\frac{\gamma}{3} t^{3}\right)\right)\right. \\
& \left.\left.-2 p q \tanh ^{2}\left(\frac{\beta}{10 s \alpha}\left(x-t y+t-\frac{\gamma}{3} t^{3}\right)\right)\right]\right\} \\
& +y \text {, } \\
& u_{3}(x, y, t) \\
& =\frac{1}{25 \alpha}\left\{3 \beta^{2}-25 \alpha-3 q \beta^{2}\right. \\
& \times\left[\operatorname{coth}\left(\frac{\beta}{10 s \alpha}\left(x-t y+t-\frac{\gamma}{3} t^{3}\right)\right)\right. \\
& \left.\left.-2 p q \operatorname{coth}^{2}\left(\frac{\beta}{10 s \alpha}\left(x-t y+t-\frac{\gamma}{3} t^{3}\right)\right)\right]\right\} \\
& +y \text {. }
\end{aligned}
$$

(see Figures 3 and 4).

Remark 2. If we direct assume that the solution of (34) can be expressed in the form

$$
F(t, \xi)=a_{0}(t)+a_{1}(t) w(\theta)+a_{2}(t) w(\theta)^{2},
$$

where $\theta=f(t) \xi+g(t), f(t)$, and $g(t)$ are continuous functions of $t$ to be determined later. $w(\theta)$ satisfies the auxiliary equation (38). Substituting (43) and (38) into (34), equating the coefficients of all powers of $w$ to zero yields a set of 


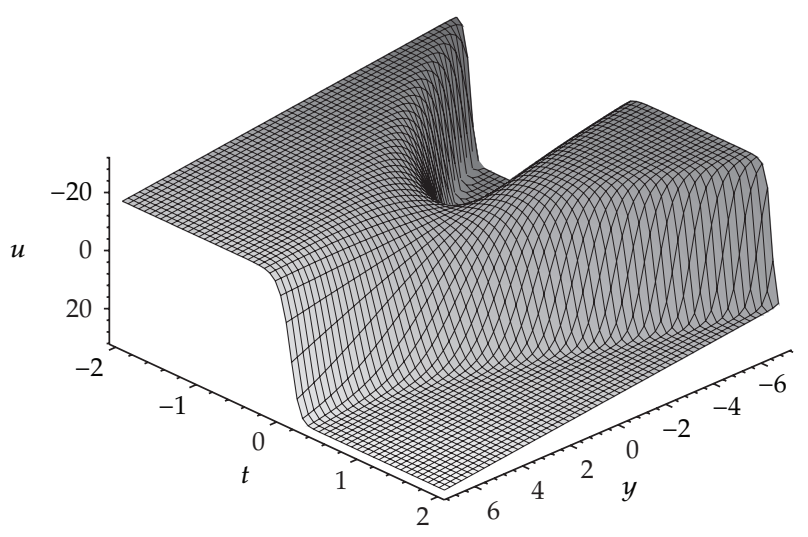

FIgURE 3: Local structure of $u_{2}(x, y, t)$ is shown as $x=1, \alpha=1, \beta=$ $10, p=-1, q=1$, and $\gamma=6$.

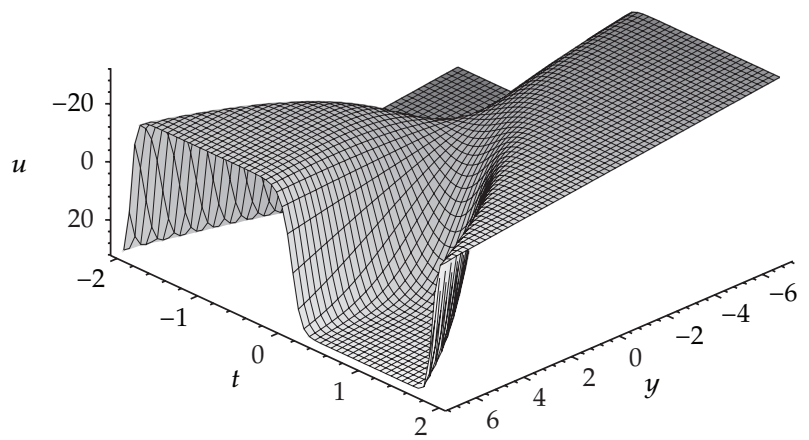

FIGURE 4: Local structure of $u_{3}(x, y, t)$ is shown as $x=1, \alpha=1, \beta=$ $10, p=-1, q=1, \gamma=6$.

function equations for $a_{0}(t), a_{1}(t), a_{2}(t), f(t)$, and $g(t)$ as follows:

$$
\begin{aligned}
w^{5}: & 2 f a_{2} q\left(12 f^{2} q^{2} \alpha+a_{2}\right)=0, \\
w^{4}: & -3 f q\left(-2 a_{1} q^{2} f^{2} \alpha+2 q f a_{2} \beta-a_{1} a_{2}\right)=0, \\
w^{3}: & -2 \beta a_{1} f^{2} q^{2}+2 a_{2}^{2} f p+40 \alpha a_{2} f^{3} q^{2} p+2 a_{2} g^{\prime} q \\
& +2 a_{0} a_{2} f q+a_{1}^{2} f q+2 a_{2} f^{\prime} \xi q+2 \gamma t^{2} a_{2} f q=0, \\
w^{2}: & -8 \beta a_{2} f^{2} p q+a_{1} g^{\prime} q+a_{2}^{\prime}+a_{0} a_{1} f q+\gamma t^{2} a_{1} f q \\
& +8 \alpha a_{1} f^{3} p q^{2}+a_{1} f^{\prime} \xi q+3 a_{1} a_{2} f q=0, \\
w^{1}: & a_{1}^{2} f p+16 \alpha a_{2} f^{3} p^{2} q+a_{1}^{\prime}+2 \gamma t^{2} a_{2} f p+2 a_{0} a_{2} f p \\
& +2 a_{2} g^{\prime} p+2 a_{2} f^{\prime} \xi p-2 \beta a_{1} f^{2} p q=0, \\
w^{0}: & a_{1} g^{\prime} p+a_{1} f^{\prime} \xi p+a_{0} a_{1} f p-2 \beta a_{2} f^{2} p^{2}+a_{0}^{\prime} \\
& +2 \alpha a_{1} f^{3} p^{2} q+\gamma t^{2} a_{1} f p=0 .
\end{aligned}
$$

Solving the system of function equations, we obtain

$$
\begin{gathered}
a_{0}(t)=\frac{3 \beta^{2}}{25 \alpha}, \quad a_{1}(t)= \pm \frac{6 \beta^{2} q}{25 s \alpha}, \\
a_{2}(t)=\frac{3 \beta^{2} q}{25 \alpha p}, \quad f(t)= \pm \frac{\beta}{10 s \alpha}, \quad g(t)=\mp \frac{\beta \gamma}{30 s \alpha} t^{3} .
\end{gathered}
$$

This result indicate the idea is equivalent to idea of Case 2 above.

Case 3. Take $k_{2}(t)=0$ and $c=1$ in (26), then

$$
\sigma=k_{1}(t) u_{x}+u_{t}-k_{1}(t) .
$$

Solving the differential equation $\sigma=0$, we obtain

$$
u=k_{1}(t)+F(\xi, y), \quad \xi=x-\int k_{1}(t) d t .
$$

Substituting (47) into (1) yield

$$
\alpha F_{\xi \xi \xi \xi}-\beta F_{\xi \xi \xi}+F F_{\xi \xi}+F_{\xi}^{2}+\gamma F_{y y}=0 .
$$

Using the transformation $F(\xi, y)=F(\eta), \eta=k \xi-c y$ and integrating the resulting equation with respect to $\eta$ we have

$$
k^{2} F^{2}+2 \gamma c^{2} F+2 k^{4} \alpha F^{\prime \prime}-2 k^{3} \beta F^{\prime}+A=0,
$$

where $A$ is an arbitrary constant, $F^{\prime}=d F / d \eta$. Suppose that the solution of ODE (49) can be expressed by a polynomial in $\left(G^{\prime} / G\right)$ as follows:

$$
F(\eta)=b_{n}\left(\frac{G^{\prime}}{G}\right)^{n}+\cdots,
$$

where $G=G(\eta)$ satisfies the second-order LODE in the form [25]

$$
G^{\prime \prime}+\lambda G^{\prime}+\mu G=0 .
$$

Balancing $F^{\prime \prime}$ with $F^{2}$ in (49) gives $n=2$. So that

$$
F(\eta)=b_{2}\left(\frac{G^{\prime}}{G}\right)^{2}+b_{1}\left(\frac{G^{\prime}}{G}\right)+b_{0}, \quad b_{2} \neq 0,
$$

where $b_{i}(i=0,1,2)$ and $\mu$ are constants to be determined later. Substituting (52) and (51) into (49). Setting these coefficients of the $G^{\prime} / G$ to zero, yields a set of algebraic equations as follows:

$$
\begin{aligned}
& k^{2} b_{2}\left(12 \alpha k^{2}+b_{2}\right)=0, \\
& 2 k^{2}\left(10 \alpha k^{2} b_{2} \lambda+b_{1} b_{2}+2 \alpha k^{2} b_{1}+2 \beta k b_{2}\right)=0, \\
& 8 \alpha k^{4} b_{2} \lambda^{2}+2 \beta k^{3} b_{1}+k^{2} b_{1}^{2}+16 \alpha k^{4} b_{2} \mu+2 k^{2} b_{2} b_{0} \\
& \quad+6 \alpha k^{4} b_{1} \lambda+4 \beta k^{3} b_{2} \lambda+2 \gamma c^{2} b_{2}=0, \\
& 2 k^{2} b_{1} b_{0}+4 \beta k^{3} b_{2} \mu+4 \alpha k^{4} b_{1} \mu+2 \gamma c^{2} b_{1}+2 \alpha k^{4} b_{1} \lambda^{2} \\
& \quad+2 \beta k^{3} b_{1} \lambda+12 \alpha k^{4} b_{2} \lambda \mu=0, \\
& 2 \gamma c^{2} b_{0}+2 \alpha k^{4} b_{1} \lambda \mu+A+2 \beta k^{3} b_{1} \mu+4 \alpha k^{4} b_{2} \mu^{2}+k^{2} b_{0}^{2}=0 .
\end{aligned}
$$


Solving the algebraic equations above yields

$$
\begin{gathered}
b_{0}=\frac{15 k^{3} \lambda \alpha(5 k \lambda \alpha+2 \beta)-3 k^{2} \beta^{2}+25 c^{2} \alpha \gamma}{25 k^{2} \alpha}, \\
b_{1}=-\frac{12 k(5 k \alpha \lambda+\beta)}{5}, \quad b_{2}=-12 k^{2} \alpha .
\end{gathered}
$$

when $25 k^{2} \alpha^{2}\left(4 \mu-\lambda^{2}\right)+\beta^{2}=0$ and $625 \alpha^{2}\left(A k^{2}-c^{2} \gamma^{2}\right)+$ $36 k^{4} \beta^{4}=0$. Consequently, we obtain the following solution of (1) for $\lambda^{2}-4 \mu>0$ :

$$
\begin{aligned}
& u_{4}(x, y, t)=-12 k^{2} \alpha \tau^{2} \\
& \times\left[\left(C_{1} \sinh \tau\left(k\left(x-\int k_{1}(t) d t\right)-c y\right)\right.\right. \\
& \left.+C_{2} \cosh \tau\left(k\left(x-\int k_{1}(t) d t\right)-c y\right)\right) \\
& \times\left(C_{1} \cosh \tau\left(k\left(x-\int k_{1}(t) d t\right)-c y\right)\right. \\
& +C_{2} \sinh \tau \\
& \left.\left.\times\left(k\left(x-\int k_{1}(t) d t\right)-c y\right)\right)^{-1}\right]^{2} \\
& +\left(12 k^{2} \lambda \alpha \tau-\frac{12 k(5 k \alpha \lambda+\beta)}{5}\right) \\
& \times\left[\left(C_{1} \sinh \tau\left(k\left(x-\int k_{1}(t) d t\right)-c y\right)\right.\right. \\
& \left.+C_{2} \cosh \tau\left(k\left(x-\int k_{1}(t) d t\right)-c y\right)\right) \\
& \times\left(C_{1} \cosh \tau\left(k\left(x-\int k_{1}(t) d t\right)-c y\right)\right. \\
& +C_{2} \sinh \tau \\
& \left.\left.\times\left(k\left(x-\int k_{1}(t) d t\right)-c y\right)\right)^{-1}\right]^{2} \\
& +\frac{15 k^{3} \lambda \alpha(5 k \lambda \alpha+2 \beta)-3 k^{2} \beta^{2}+25 c^{2} \alpha \gamma}{25 k^{2} \alpha} \\
& +\frac{\lambda^{2}}{4}
\end{aligned}
$$

where $\tau=(1 / 2) \sqrt{\lambda^{2}-4 \mu}$.

\section{Conclusions}

Based on the fact that the periodic solution of $(2+1) \mathrm{D} \mathrm{KdV}$ Burgers equation does not exist, we have obtained Saddlenode Heteroclinic Orbits. By applying the Lie group method, we reduce the $(2+1) \mathrm{D} \mathrm{KdV}$ Burgers equation to (1+1)-dimensional equations including the $(1+1)$-dimensional linear partial differential equation with constants coefficients (29), (48) and (1+1)-dimensional nonlinear partial differential equation with variable coefficients (34). By solving the equations (29), (34), and (48), we obtain some new exact solutions and discover the strange phenomenon of sympathetic vibration to evolution of nontraveling wave soliton for the $(2+1) \mathrm{D} \mathrm{KdV}$ Burgers equation. Our results show that the unite of Lie group method with others is effective to search simultaneously exact solutions for nonlinear evolution equations. Other structures of solutions with symmetry (26) are to be further studied.

\section{Acknowledgments}

The authors would like to thank professor S. Y. Lou for the helpful discussions. This work was supported by key research projects of Sichuan Provincial Educational Administration no. 10ZA021 and Chinese Natural Science Foundation Grant no. 10971169.

\section{References}

[1] R. S. Johnson, "A non-linear equation incorporating damping and dispersion," Journal of Fluid Mechanics, vol. 42, pp. 49-60, 1970.

[2] L. van Wijngaarden, "One-dimensional flow of liquids containing small gas bubbles," Annual Review of Fluid Mechanics, vol. 4, pp. 369-396, 1972.

[3] G. Gao, "A theory of interaction between dissipation and dispersion of turbulence," Scientia Sinica A, vol. 28, no. 6, pp. 616-627, 1985.

[4] W. X. Ma, "An exact solution to two-dimensional Korteweg-de Vries-Burgers equation," Journal of Physics A, vol. 26, no. 1, pp. L17-L20, 1993.

[5] Z. B. Li and M. L. Wang, "Travelling wave solutions to the twodimensional KdV-Burgers equation," Journal of Physics A, vol. 26, no. 21, pp. 6027-6031, 1993.

[6] J. Zhang, "Soliton-like solutions for the $(2+1)$-dimensional nonlinear evolution equation," Communications in Theoretical Physics, vol. 32, no. 2, pp. 315-318, 1999.

[7] E. G. Fan and H. Q. Zhang, "A note on the homogeneous balance method," Physics Letters A, vol. 246, pp. 403-406, 1998.

[8] S. Y. Lou and G. J. Ni, "The relations among a special type of solutions in some $(D+1)$-dimensional nonlinear equations," Journal of Mathematical Physics, vol. 30, no. 7, pp. 1614-1620, 1989.

[9] X.-Y. Tang and S.-Y. Lou, "Folded solitary waves and foldons in $(2+1)$ dimensions," Communications in Theoretical Physics, vol. 40, no. 1, pp. 62-66, 2003.

[10] E. J. Parkes and B. R. Duffy, “Travelling solitary wave solutions to a compound KdV-Burgers equation," Physics Letters A, vol. 229, no. 4, pp. 217-220, 1997.

[11] A. Elgarayhi and A. Elhanbaly, "New exact traveling wave solutions for the two-dimensional KdV-Burgers and Boussinesq equations," Physics Letters A, vol. 343, no. 1-3, pp. 85-89, 2005.

[12] A. Molabahrami, F. Khani, and S. Hamedi-Nezhad, "Soliton solutions of the two-dimensional KdV-Burgers equation by homotopy perturbation method," Physics Letters A, vol. 370, no. 5-6, pp. 433-436, 2007.

[13] H. L. Chen and D. Q. Xian, "Periodic wave solutions for the Klein-Gordon-Zakharov equations," Acta Mathematicae Applicatae Sinica. Yingyong Shuxue Xuebao, vol. 29, no. 6, pp. 1139-1144, 2006. 
[14] D. Q. Xian, "New exact solutions to a class of nonlinear wave equations," Journal of University of Electronic Science and Technology of China, vol. 35, no. 6, pp. 977-980, 2006.

[15] D.-Q. Xian and H.-L. Chen, "Symmetry reduced and new exact non-traveling wave solutions of potential KadomtsevPetviashvili equation with p-power," Applied Mathematics and Computation, vol. 217, no. 4, pp. 1340-1349, 2010.

[16] J.-H. He, "Periodic solutions and bifurcations of delay-differential equations," Physics Letters A, vol. 347, no. 4-6, pp. 228-230, 2005.

[17] Z. Dai and D. Xian, "Homoclinic breather-wave solutions for Sine-Gordon equation," Communications in Nonlinear Science and Numerical Simulation, vol. 14, pp. 3292-3295, 2009.

[18] Z. Dai, D. Xian, and D. Li, "Homoclinic breather-wave with convective effect for the $(1+1)$ - dimensional boussinesq equation," Chinese Physics Letters, vol. 26, no. 4, Article ID 040203, 2009.

[19] Z. Dai, Z. Li, Z. Liu, and D. Li, "Exact homoclinic wave and soliton solutions for the 2D Ginzburg-Landau equation," Physics Letters A, vol. 372, no. 17, pp. 3010-3014, 2008.

[20] D. Xian and Z. Dai, "Application of exp-function method to potential kadomtsev-petviashvili equation," Chaos, Solitons and Fractals, vol. 42, pp. 2653-2659, 2009.

[21] G.-C. Xiao, D.-Q. Xian, and X.-Q. Liu, "Application of expfunction method to Dullin-Gottwald-Holm equation," Applied Mathematics and Computation, vol. 210, no. 2, pp. 536-541, 2009.

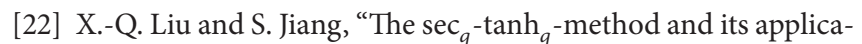
tions," Physics Letters A, vol. 298, no. 4, pp. 253-258, 2002.

[23] S. K. Liu and S. D. Liu, Solitary wave and turbulence, Shanghai Scientifc and Technological Education, Shanghai, China, 1994.

[24] S. K. Liu and S. D. Liu, Nonliner Equations in Physics, Peking University, Beijing, China, 2000.

[25] M. Wang, X. Li, and J. Zhang, "The $\left(G^{\prime} / G\right)$-expansion method and travelling wave solutions of nonlinear evolution equations in mathematical physics," Physics Letters A, vol. 372, no. 4, pp. 417-423, 2008. 


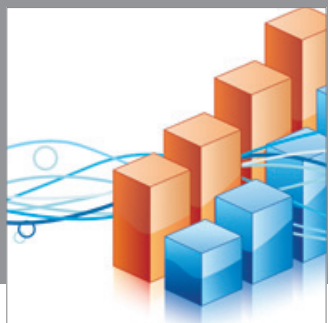

Advances in

Operations Research

mansans

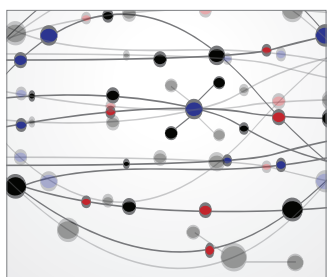

The Scientific World Journal
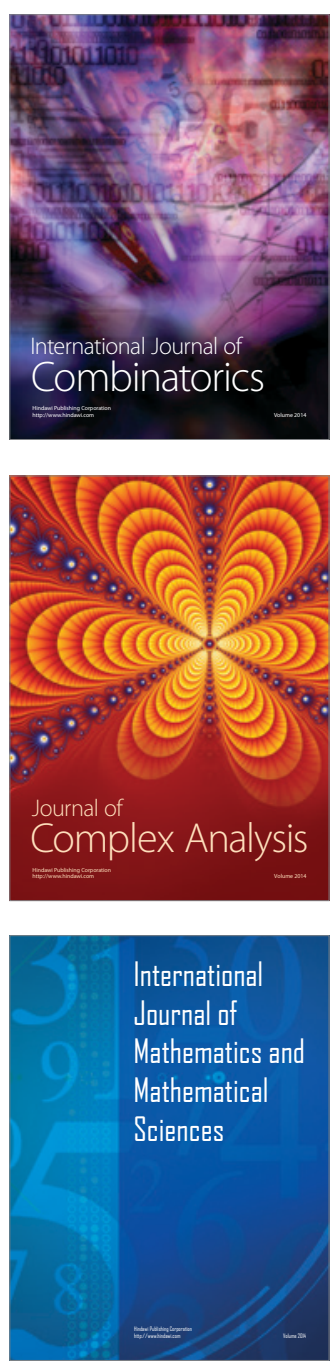
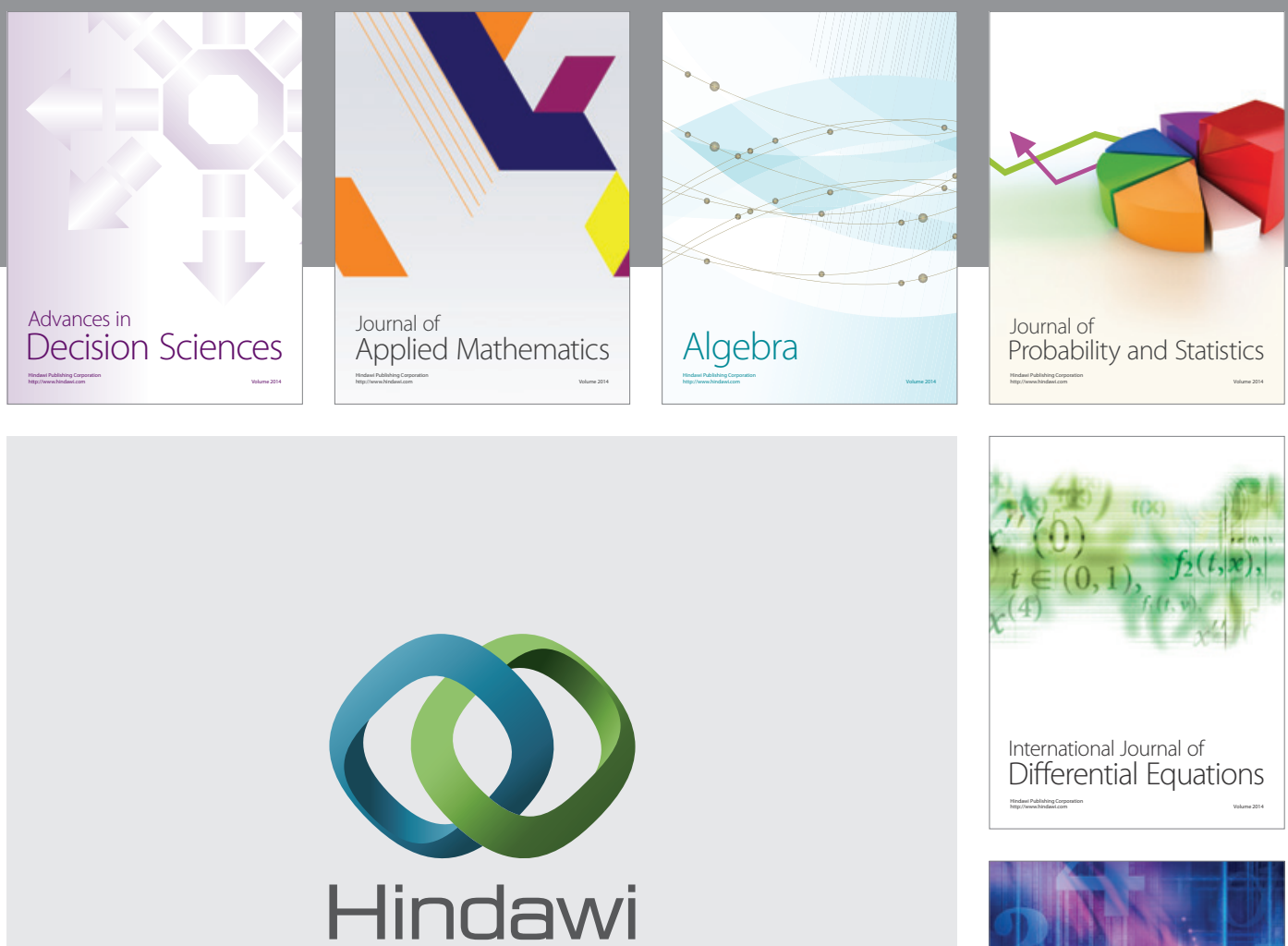

Submit your manuscripts at http://www.hindawi.com
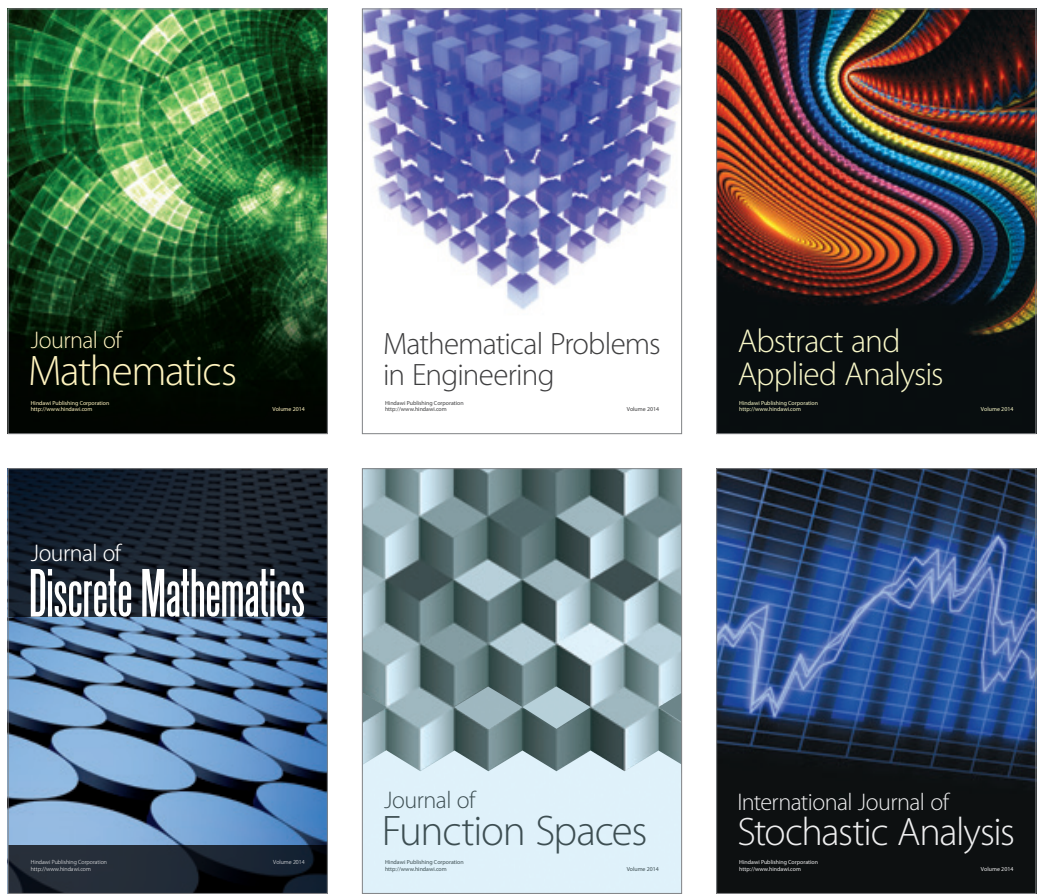

Journal of

Function Spaces

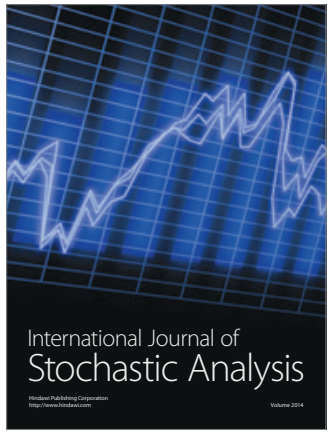

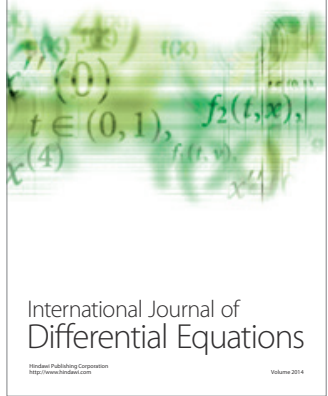
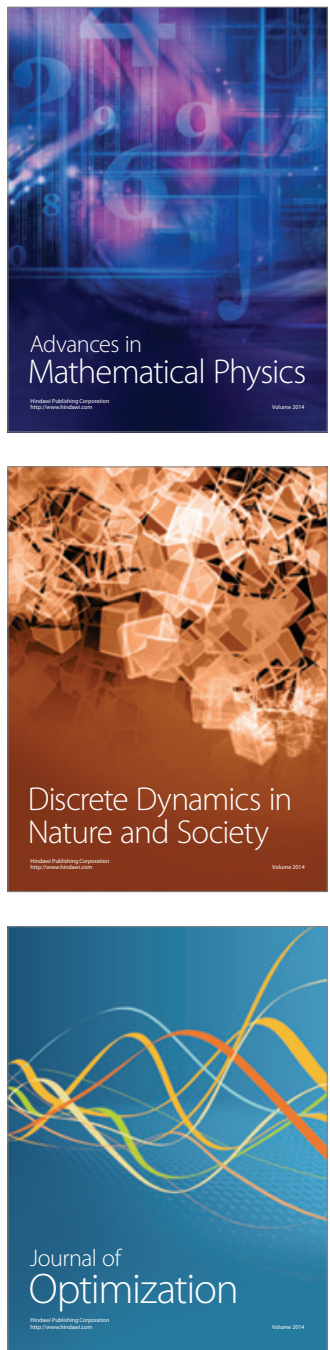